\title{
Fridays for Future als Lern- und Erfahrungsraum: Befunde zu den Beteiligungsformaten, den Motiven und der Selbstwirksamkeitserwartung von Engagierten
}

\begin{abstract}
Zusammenfassung
In diesem Beitrag wird das Engagement für die Fridays for Future (FFF)-Bewegung aus bildungswissenschaftlicher Perspektive in den Blick genommen. Aktives Engagement für die FFF-Bewegung wird als Lern- und Erfahrungsraum verstanden und ausgewählte Befunde einer Online-Befragung ( $n=194)$ vorgestellt. Zum einen werden spezifische Charakteristika der FFF-Bewegung mit Blick auf Lern- und Bildungsprozesse skizziert, zum anderen empirische Analysen zu verschiedenen Beteiligungsmöglichkeiten dargestellt. Dabei wird die Vielfalt der Beteiligungsformate und die Motivation der Engagierten näher betrachtet und danach gefragt, ob sich je nach Beteiligungsform Unterschiede in den Motiven und der Selbstwirksamkeitserwartung finden lassen. Die Ergebnisse werden weiterführend mit Blick auf potenzielle Lernerfahrungen diskutiert.
\end{abstract}

Schlüsselworte: freiwilliges Engagement; Fridays for Future; Motive; Selbstwirksamkeitserwartung; Lernumwelten

\begin{abstract}
In this article, engagement in the Fridays for Future (FFF) movement is examined from an educational point of view. Framing active engagement in the FFF movement theoretically as a learning opportunity, selected findings from an online survey $(n=194)$ are presented. In a theoretical perspective, specific characteristics of the FFF movement are outlined with regard to learning and educational processes. Starting point of the empirical analyses are different possibilities of participation. On the one hand, an insight into the various participation formats is given. On the other hand, it is asked what motivates those involved to work for sustainable development and whether differences in motives and self-efficacy can be found depending on the form of participation. The results will be linked back to the theoretical frame of reference and discussed further.
\end{abstract}

Keywords: Volunteering; Fridays for Future; Motives; Self-efficacy Expectations; Learning Environments
Die Proteste für eine zukunftsorientierte (Klima-)Politik brachten vor der Pandemie zuletzt tausende von Menschen in zahlreichen Ländern auf die Straße. Unter dem Slogan „Fridays for Future“ (FFF) setzt sich die jüngere Generation für eine neue bzw. andere Klimapolitik ein. Es scheint, als hätten insbesondere Schülerinnen und Schüler sowie Studierende in der Protestbewegung eine eigene Form aktiver gesellschaftlicher Partizipation gefunden, die in öffentlichen Diskursen z. T. auch kritisch kommentiert wird (vgl. Reuter, 2020; Haunss, Sommer \& Fritz, 2020; Teune, 2020). Mittlerweile liegen erste wissenschaftliche Ergebnisse zu den konkreten Handlungsmotivationen der Teilnehmenden aus der Protest- und Bewegungsforschung vor, welche die Behauptung, dass die Teilnehmenden vorwiegend an den Demonstrationen partizipieren, um nicht am (Schul-)Unterricht teilzunehmen, deutlich entkräften (vgl. Wahlström et al., 2019; Koos \& Lauth, 2019). Die Studien zum Themenfeld sind bislang auf die exemplarische Analyse der Proteste in einzelnen (Groß-)Städten begrenzt und vorwiegend auf die Befragung von Teilnehmenden an den Demonstrationen fokussiert.

In diesem Beitrag wird die Perspektive erweitert, in dem das Engagement der Jugendlichen für FFF in seiner Vielfältigkeit näher in den Blick genommen und als Lernund Erfahrungsraum gerahmt wird. Betrachtet werden nicht nur Teilnehmende an den Demonstrationen, sondern auch diejenigen, die sich aktiv für FFF einsetzen (z.B. in Ortsgruppen). Es werden dazu ausgewählte Befunde einer Online-Befragung ( $\mathrm{n}=194)$ vorgestellt. Um die spezifischen Charakteristika der Bewegung mit Blick auf potenzielle Lern- und Bildungsprozesse herauszuarbeiten, wird zunächst ein theoretischer Referenzrahmen aufgespannt. Es folgt eine Einführung in den Forschungsstand, die Fragestellungen und eine Beschreibung der durchgeführten Studie. Anschließend werden ausgewählte Ergebnisse zu den Beteiligungsformaten vorgestellt und diskutiert. 


\section{Fridays for Future als Lern- und Erfahrungsraum}

Freiwilliges Engagement für die FFF-Bewegung, welches über eine reine Teilnahme an Demonstrationen hinausgeht, wird in diesem Beitrag als informeller Lern- und Erfahrungsraum verstanden. Nationale und internationale Engagementsforschung zeigt, dass dem freiwilligen Engagement im Allgemeinen weitreichende Effekte zugeschrieben werden (z.B. Braun, 2006; Duguid et al., 2013; Düx, 2018; Elsdon et al., 2002). Verschiedene Studien untersuchen freiwilliges Engagement als Lernund Entwicklungsraum (z.B. Benedetti, 2015; Elsdon et al., 2002; Hansen, 2008) und das Lernen bzw. die (Kompetenz-) Entwicklung innerhalb der Tätigkeiten (z.B. Düx et al., 2009; Göring und Mutz, 2016; Reinders, 2014). Empirisch zeigt sich u.a., dass freiwilliges Engagement die Entwicklung personaler, sozialer, kultureller und instrumenteller Kompetenzen anregt und als ein bedeutender Lernraum, jenseits von Familie und formalen Lernorten, beschrieben werden kann (vgl. Düx et al., 2009). Für die FFF-Bewegung lassen sich spezifische Charakteristika beschreiben, die im Folgenden in der Perspektive auf Lern- und Bildungsprozesse skizziert werden.

\section{Glokale Bewegung}

Die FFF-Bewegung führt eine global-lokale Verschränkung eindrücklich vor Augen. Als globaler Klimastreik, der lokal in Ortsgruppen organsiert wird, zeigt sich sowohl im Gegenstand der Proteste selbst als auch in der Netzwerkstruktur der Bewegung die Verwobenheit von globalen und lokalen Prozessen. Für Lern- und Bildungsprozesse im Horizont weltgesellschaftlicher Bildung kann dies die Chance bieten glokale Raumbezüge (Robertson 1998, S. 208) sicht- und erfahrbar zu machen, z.B. durch die Entwicklung kollektiver Identitäten lokaler Ortsgruppen in Aushandlung und Abgrenzung zu deutschlandweiten und globalen Gruppierungen (Döninghaus et al., 2020).

\section{Schulstreik im öffentlichen Diskurs}

Da die FFF-Proteste als Schulstreik gerahmt sind und damit explizit in Konflikt mit der Schulpflicht stehen, werden für Schülerinnen und Schüler Aushandlungsprozesse bedeutsam. In der Geschichte stellen Schulstreiks zwar kein Novum dar, mit Blick auf klimapolitische Proteste sind sie allerdings eine neue Aktionsform (Rucht \& Rink, 2020). Besonders zeichnet die FFF-Bewegung die anhaltende Mobilisierung einer großen Anzahl von Kindern und Jugendlichen aus (Teune, 2020), wodurch ein breiter öffentlicher Diskurs bezüglich des Umgangs mit den Schulstreiks angeregt wurde (Hanschmann, 2019; Goldenbaum \& Thompson, 2020). Teilnehmende an den Protesten wie auch Engagierte sind dadurch herausgefordert, ihre Teilnahme zu legitimieren und zu begründen. Da FFF einen Generationenkonflikt proklamiert, werden zudem explizit auch intragenerationale Aushandlungsprozesse angeregt. In der Perspektive auf Lern- und Bildungsprozesse kann der breite öffentliche Diskurs dazu bewegen Stellung zu beziehen und sich intensiv mit der Dringlichkeit des Themas, wissenschaftlichen Grundlagen sowie den eigenen Rechten auseinanderzusetzen.

\section{Gelebte Demokratie}

Grundsätzlich stellen die Proteste selbst ein Mittel zur Artikulation bislang vernachlässigter Interessen und Themen dar und sind damit wesentlicher Bestandteil von Demokratie (Sommer et al., 2020). Die basisdemokratische Ausrichtung der Bewegung (Rucht \& Rink, 2020) ermöglicht darüber hinaus im aktiven Engagement für FFF die Chance, in den lokalen Ortsgruppen gemeinsam Konsens zu verhandeln und demokratische Entscheidungsfindungen zu erleben. Die unterschiedlichen biografischen Hintergründe und Kenntnisse der Engagierten können dabei als Potenzial für Lern- und Bildungsprozesse im Sinne einer „Tradierung lebensweltlicher Kompetenzen und Werte“ (Düx \& Sass, 2005) interpretiert werden.

\section{Spontane Verstetigung}

In Reaktion auf den zunehmenden Koordinations- und Organisationsaufwand bildeten sich in der Protestbewegung spezifische Arbeitsgruppen, die sich mit Bereichen wie der (überregionalen) internen Kommunikation, Struktur, Finanzen, Demo-Vorbereitungen usw. auseinandersetzen (Ruch \& Rink, 2020). Mit Blick auf Lern- und Bildungsprozesse ermöglicht diese Ausdifferenzierung und Professionalisierung der Bewegung die Chance, direkt an dem Aufbau von Netzwerkstrukturen und Organisations- sowie Arbeitseinheiten zu partizipieren und eigene Stärken gezielt einzubringen und Verantwortung zu übernehmen.

\section{Digitale Vernetzung}

Die FFF-Bewegung führt die Bedeutung digitaler Medien für freiwilliges Engagement deutlich vor Augen, denn die Möglichkeit des weltweiten digitalen Informationsaustauschs hat vermutlich maßgeblich zum Aufbau der globalen Bewegung beigetragen. Das Internet bietet die Möglichkeit, sich orts- und zeitunabhängig zu vernetzen und in Austausch zu treten. Digitale Medien können Arbeitsprozesse (z.B. durch Projektmanagementsoftware) unterstützen und beschleunigen, helfen ortsübergreifende Strukturen zu etablieren und z.B. über soziale Medien Informationen schnell und verhältnismäßig barrierefrei an viele Menschen weiterzugeben. Als Digital Natives ist gerade die junge Generation mit den Potenzialen des Internets vertraut (vgl. Hurrelmann \& Albrecht, 2020). Das Engagement in der digital vernetzten Bewegung kann die Chance bieten digitale Kompetenzen auszubauen, neue Formen des Umgangs sowie vielfältige Unterstützungstools kennen zu lernen und anwenden zu können.

\section{Kreative Gestaltungspotenziale}

Ein Merkmal, welches bei der FFF-Bewegung im Vergleich mit anderen Protesten deutlich hervorsticht, ist die Kreativität der Proteste (Teune, 2020). Hinter den vielseitigen und bunten Schildern, den mitreißenden Parolen, Musik mit politischen Botschaften steht ein besonderer Weltzugang, der gerade vor dem Hintergrund kultureller Lern- und Bildungsprozesse an Relevanz gewinnt. In Diskursen kultureller Bildung wird der kreativen Erschließung von Welt ein besonderes Potenzial für 
Lern- und Bildungsprozesse im Kontext einer nachhaltigen Entwicklung zugesprochen (z.B. Braun-Wanke \& Wagner, 2020). Eine nachhaltige Entwicklung bedarf einer kreativen Gestaltungskompetenz, die auch eine sinnlich-ästhetische Dimension einschließt.

\section{Forschungsstand und Fragestellungen Individuelle Ebene: Merkmale der FFF-Teilnehmenden}

Bislang lassen sich auf Basis von einzelnen Befragungen von Protestierenden in ausgewählten Städten erste Ergebnisse zu den Merkmalen der Teilnehmenden an der FFF-Bewegung skizzieren (Sommer et al., 2020; Koos \& Lauth, 2020; Willis $\&$ Loy, 2021). Die bisherigen Studien zeigen, dass die Demonstrationen in der frühen Phase der Bewegung insbesondere von jungen, gut gebildeten Menschen besucht wurden. Frauen sind überrepräsentiert und nehmen in der Bewegung einen zentralen Stellenwert ein (Sommer et al., 2020). Die Altersstruktur veränderte sich im Zeitverlauf dahingehend, dass zunehmend ältere Personen an den Protesten partizipieren (Koos \& Lauth, 2020; Sommer et al., 2020). Mit Blick auf ihre politische Ausrichtung sind die Demonstrierenden überwiegend links eingestellt und zeigen ein relativ hohes und stabiles Vertrauen in demokratische Institutionen (Sommer et al., 2020). Zu den Antrieben von jungen Menschen, sich für die Umwelt einzusetzen, arbeiten Willis \& Loy (2021) die Beteiligung von Freunden, die Identifikation mit anderen Engagierten und persönliche Normen und Werte als stärkste Prädiktoren für das aktive Engagement heraus (Willis \& Loy, 2021).

\section{Strukturelle Ebene: Die FFF-Bewegung}

Rucht \& Rink (2020) geben auf Basis von Interviews mit Engagierten erste Einblicke in die Strukturen der FFF-Bewegung. Auch wenn die Bewegung in ihren Anfängen günstige Voraussetzungen für die Informations- und Öffentlichkeitsarbeit vorfand, bedeutete die logistische Arbeit mit zunehmender Größe einen massiven Zeitaufwand für die Aktivistinnen und Aktivisten. Hervorgehoben wird die Unterstützung durch bestehende Gruppierungen (wie z.B. Greenpeace, BUND usw.) und die breiten Vorerfahrungen und Kenntnisse der Engagierten, die zumeist bereits zuvor in Klima- und Umweltgruppen aktiv waren. Die Strukturierungsarbeit (z.B. die Entwicklung von Kommunikationsstrukturen, Arbeitsteilung, Entscheidungsfindungsprozesse und mediale Repräsentation) wird als (zeit-)aufwendiger (Arbeits-)Bereich beschrieben. Döninghaus et al. (2020) verweisen auf eine konsensdemokratische Gruppenstruktur, welche durch asymmetrische Redeverteilungen und bestehende Wissenshierarchien eingeschränkt wird. Die untersuchten Ortsgruppen weisen hierzu einen bewussten und reflexiven Zugang auf (Döninghaus et al., 2020).

\section{Forschungsdesiderate und Fragestellungen}

Insgesamt lässt sich feststellen, dass die empirischen Analysen vorwiegend in der Protest- und Bewegungsforschung verortet sind. Aus bildungs- und erziehungswissenschaftlicher Perspektive finden sich erste theoretische Annäherungen (vgl. Budde,
2020; Hellgermann, 2020; Holz et al. 2021), ein empirischer Zugang zum Feld fehlt bislang. Daran anknüpfend werden in diesem Beitrag ausgewählte Befunde vorgestellt, die verschiedene Beteiligungsformen in den Mittelpunkt stellen.

In den Analysen wird (1) nach verschiedenen Formen der Beteiligung an der FFF-Bewegung gefragt, die einen Einblick in die vielfältigen Erfahrungen innerhalb des Engagements ermöglichen. In den weiterführenden Analysen wird mit Blick auf die Beteiligungsform zwischen Personen unterschieden, die ausschließlich an den Demonstrationen teilnehmen und Personen, die darüber hinaus bei FFF aktiv sind und z.B. Organisationsarbeit im Hintergrund leisten oder Reden halten.

Es werden (2) Motive der Teilnehmenden dargestellt und Unterschiede in den Motivationen der Teilnehmenden in Abhängigkeit der Beteiligungsform untersucht, da aus lerntheoretischer Perspektive angenommen wird, dass Motive den Lernerfolg maßgeblich beeinflussen (z.B. Deci \& Ryan, 1993).

Im nächsten Schritt wird danach gefragt, ob sich (3) Unterschiede in der Selbstwirksamkeitserwartung der Engagierten in Abhängigkeit der Beteiligungsformate finden lassen. Eine hohe Selbstwirksamkeitserwartung kann als eine Grundvoraussetzung für Handeln und damit auch für gesellschaftlichen Engagement beschrieben werden. Diese kann demnach in einem Zusammenhang mit einer mehr oder weniger intensiven Beteiligung an der Bewegung stehen (z.B. Bandura, 1997). Positive Korrelationen zwischen der Selbstwirksamkeitserwartung und der Intention, sich aktiv für die Umwelt einzusetzen, ließen sich bereits identifizieren (z.B. Reese, 2020). Es zeigt sich auch, dass die Selbstwirksamkeitserwartung ein Prädiktor von Umweltaktivismus sein kann (Lee et al., 2014; Wallis \& Loy, 2021). Während bislang nicht klar zwischen einer reinen Teilnahme an Demonstrationen und dem aktiven Engagement differenziert wurde, wird in diesem Beitrag die Selbstwirksamkeitserwartung in Abhängigkeit verschiedener Beteiligungsformate betrachtet. Aus bildungswissenschaftlicher Perspektive sind die Unterschiede in der Selbstwirksamkeitserwartung verschiedener Beteiligungsgruppen interessant, da sie auf mögliche Lern- und Erfahrungsprozesse verweisen können.

\section{Daten, Stichprobe \& Erhebungsinstrumente}

Daten: Die hier vorgestellten Ergebnisse basieren auf einer Online-Befragung $(\mathrm{n}=194)$ von Demonstrationsteilnehmenden $(n=39)$ als auch Personen, die sich darüber hinaus aktiv für die FFF-Bewegung engagieren $(n=103)$. Es nahmen auch 29 Personen an der Umfrage teil, die sich nicht an FFF beteiligen.

Stichprobe: Die vorliegende Stichprobe setzt sich aus Schülerinnen und Schülern (49,7 \%), Studierenden (26,67\%), Auszubildenden (3,64\%), Berufstätigen (12,12\%) und Sonstigen $(7,88 \%)$ zusammen. Die Teilnehmenden waren durchschnittlich 21 Jahre alt $(\mathrm{Min}=16, \mathrm{Max}=62)^{1}$. In der vorliegenden Stichprobe sind Frauen mit 60,82 \% leicht überrepräsentiert. Die Teilnehmenden stammen überwiegend aus gut gebildeten Elternhäusern.

Erhebungsinhalte: ${ }^{2}$ Die Form der Beteiligung wurde differenziert erfasst und reicht von einer reinen Teilnahme an Demonstration(en) bis hin zum Engagement in Ortsgruppen, internationaler Vernetzung und der Organisation von Internetkampagnen. Die Teilnehmenden konnten ihre Tätigkeit darü- 
ber hinaus näher beschreiben. Für die Abfrage von Motiven wurde, neben einer offenen Abfrage, eine vierstufige Likert-Skala eingesetzt. Theoretisch wird zwischen drei Dimensionen unterschieden, die sich auch faktorenanalytisch abbilden lassen: soziale, selbstbezogene und gesellschaftliche Motive. Die verwendeten Items sind z. T. aus bestehenden Studien übernommen (Sommer et al., 2020; Alberts et al., 2002) und wurden sprachlich angepasst.

Für die Erhebung der allgemeinen Selbstwirksamkeitserwartung (ASWE) wurde das deutschsprachige Instrument von Schwarzer und Jerusalem (1999) eingesetzt, welches auf den theoretischen Grundlagen Banduras basiert (Bandura, 1997). Die Skala misst die subjektive Überzeugung, problematische und kritische Situationen aus eigener Kraft und Anstrengung erfolgreich bewältigen zu können (Schwarzer \& Jerusalem, 2002). In der vorliegenden Stichprobe liegt die interne Konsistenz (Cronbachs alpha) der eindimensionalen Skala bei .82, womit ein ähnlich hoher Wert wie in anderen deutschen Stichproben erzielt werden konnte (Schwarzer \& Jerusalem, 1999).

\section{Ausgewählte Ergebnisse \\ Formen der Beteiligung}

In der vorliegenden Studie wurde explizit nach verschiedenen Formen der Beteiligung gefragt (vgl. Abb. 1). Die Ergebnisse verweisen auf ein vielseitiges Engagement der Befragten, welches häufig über die reine Teilnahme an Demonstrationen hinausreicht.

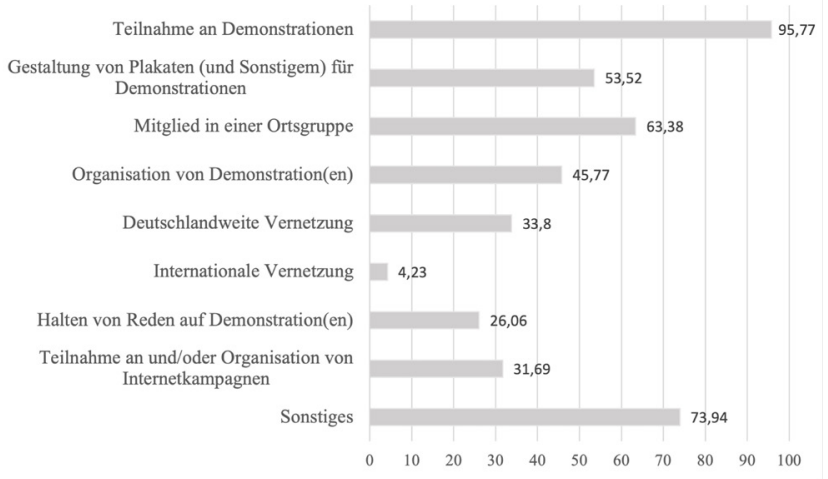

Abb. 1: Formate der Beteiligung in \% (Mehrfachangaben möglich); Quelle: eigene Darstellung

Das Engagement spielt sich überwiegend in den Ortsgruppen $a b$, wobei die Organisation der Demonstrationen neben der Gestaltung von Plakaten (und Sonstigem) für die Demonstrationen einen zentralen Stellenwert einnimmt. Das Engagement der Befragten umfasst nach eigenen Angaben darüber hinaus vor allem Öffentlichkeitsarbeit (z.B. Vorträge, Workshops, Pressekonferenzen, Livestreams) sowie die Kommunikation mit und die Vernetzung von verschiedenen lokalen Akteuren (wie z.B. (Kommunal-)Politik, Parents for Future, Umweltgruppen). Gerade von Studierenden wird das Hineinwirken in die Hochschule thematisiert (z.B. in Form von Forderungen an die Hochschulleitung, Vernetzung von transdisziplinärer Wissenschaft, PublicClimateSchool).

Mit Blick auf die eingangs skizzierten Lern- und Erfahrungspotenziale der Bewegung lässt sich festhalten, dass die internationale Vernetzung in der konkreten Arbeit eine weniger große Rolle spielt als angenommen. Dies spiegelt sich darin wider, dass lediglich 4,3\% der Engagierten angeben, sich mit internationaler Netzwerkarbeit zu beschäftigen. Zudem wird deutlich, dass die ehemals spontane Protestbewegung mittlerweile strukturell ausdifferenziert und in lokalen Arbeitsgruppen organisiert wird. Die Antworten der Engagierten deuten darauf hin, dass der überwiegende Teil der Engagierten in die Presse- und Öffentlichkeitsarbeit eingebunden ist, wohingegen organisatorische Arbeiten (z.B. Verwaltung von Finanzen) auf wenige Einzelpersonen verteilt ist.

\section{Motive in Abhängigkeit der Beteiligungsform}

Für die Darstellung der Motive wurden die beiden Zustimmungskategorien („trifft völlig zu“ und „trifft eher zu“) zusammengefasst. Wie in Abbildung 2 ersichtlich, erzielen gesellschaftliche Motive die höchste Zustimmung. Die FFF-Bewegung wird also als eine Möglichkeit wahrgenommen, sich aktiv für gesellschaftliche Veränderungsprozesse einzusetzen und Einfluss auf politische Entscheidungsprozesse zu nehmen.

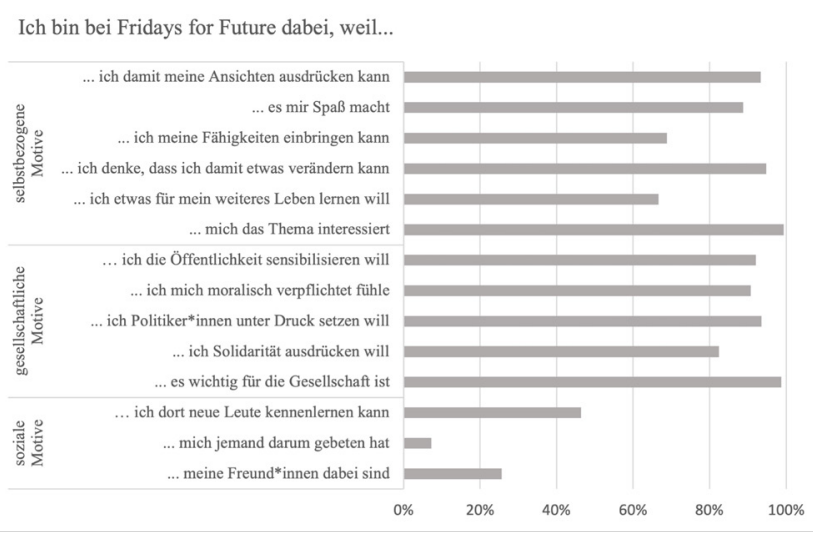

Abb. 2: Motive der Teilnehmenden (prozentuale Darstellung der zusammengefassten Zustimmungskategorien); Quelle: eigene Darstellung

Im nächsten Schritt wird der Zusammenhang zwischen intrinsischer Motivation und Beteiligungsform überprüft, indem aus den intrinsischen bzw. selbstgezogenen Motiven ein Summenscore [6-24] gebildet wird. Unterschieden wird zwischen Personen, die ausschließlich an Demonstrationen teilnehmen und/oder Plakate dafür gestalten, und Personen, die sich darüber hinaus aktiv für FFF engagieren. Diese Differenzierung ist aus bildungswissenschaftlicher Sicht insofern interessant, als dass angenommen werden kann, dass eine höhere intrinsische Motivation einen fruchtbaren Boden für Lern- und Bildungsprozesse darstellt. Gerade selbstbestimmte und autonome Handlungen, die den eigenen Zielen und Wünschen entsprechen (also intrinsisch motiviert sind) münden in bessere Leistungen, effektiveres Lernen und positive Affekte (Deci \& Ryan, 1993) und stellen damit eine optimale Ausgangssituation für Lernprozesse im Rahmen des aktiven Engagements dar.

Es zeigt sich, dass Personen, die ein hohes Engagement aufweisen, höhere Werte in der intrinsischen Motivation ausweise als Personen, die an Demonstrationen teilnehmen und/oder Plakate hierfür gestalten (vgl. Abb. 3). Die Mittel- 
werte der Gruppe der hoch Engagierten sind signifikant höher als die Mittelwerte derjenigen, die lediglich an den Protesten teilnehmen $(\mathrm{t}(132)=-2.75, \mathrm{p}=0,00)$. Dies ist insofern interessant, als dass in Studien zu den Beweggründen für Umweltengagement bislang insbesondere weniger selbstbestimmte Motive (wie z.B. die Teilnahme von Freundinnen und Freunden und Identifikation mit Anderen) als starke Prädiktoren beschrieben werden (Wallis \& Loy, 2021). Aus bildungswissenschaftlicher Perspektive kann die höhere intrinsische Motivation an dieser Stelle als wichtige Bedingung für Lern- und Bildungsprozesse interpretiert werden.

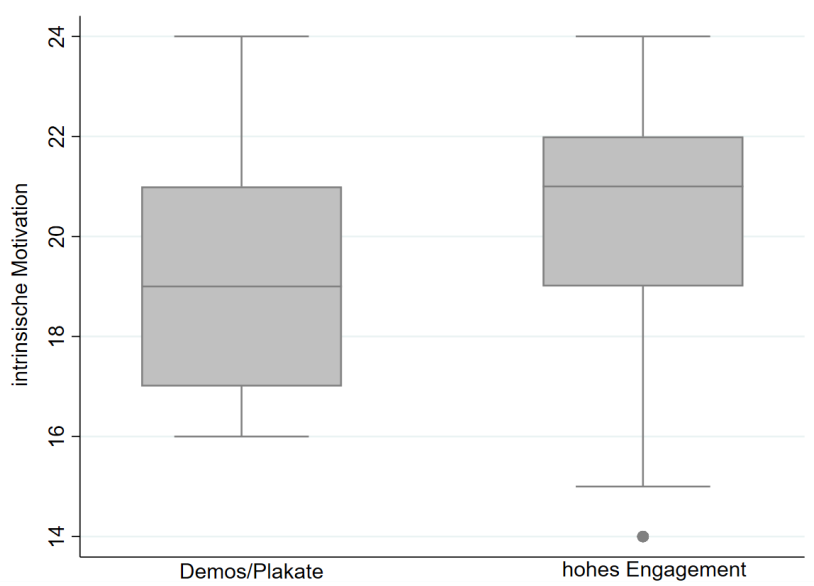

Abb. 3: Intrinsische Motivation differenziert nach Beteiligungsgruppen; Quelle: eigene Darstellung

\section{Selbstwirksamkeitserwartung in Abhängigkeit der Beteiligungsform}

Um zu überprüfen, ob sich die Engagierten mit Blick auf ihre Selbstwirksamkeitserwartung voneinander unterscheiden, wurde in den vorliegenden Analysen zwischen drei (Vergleichs-) Gruppen unterschieden: Personen, 1) die sich nicht an FFF beteiligen, 2) die lediglich an Demonstrationen teilnehmen und/oder Plakate dafür gestalten, 3) Personen, deren Engagement über eine reine Teilnahme an Demonstrationen hinausreicht (z.B. Beteiligung an einer Ortsgruppe).

Es zeigt sich, dass Personen, die sich nicht an FFF beteiligen im Durchschnitt die niedrigste allgemeine Selbstwirksamkeitserwartung aufweisen. Die höchste Selbstwirksamkeitserwartung zeigen Personen, die an den Demonstrationen teilnehmen und/oder Plakate dafür gestalten. Junge Menschen, die sich über die Demonstrationen hinaus aktiv bei FFF einbringen, haben eine niedrigere Selbstwirksamkeitserwartung als die Teilnehmenden an Demonstrationen (vgl. Abb. 4). Die Gruppenunterschiede wurden mit Hilfe eines Kruskal-Wallis H-Test überprüft und erwei- sen sich als statistisch signifikant $(X 2(2)=7,081, \mathrm{p}=0,029)$.

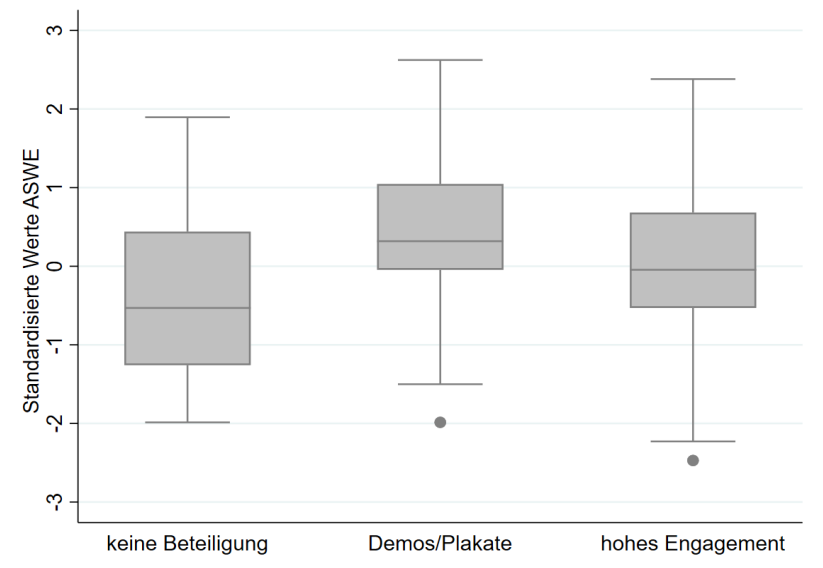

Abb. 4: Allgemeine Selbstwirksamkeitserwartung (ASWE) differenziert nach Beteiligungsgruppen; Quelle: eigene Darstellung

\section{Diskussion}

Die vorliegende theoretische und empirische Annäherung an das Themenfeld ermöglicht eine erste Perspektive auf die FFF-Bewegung als Lern- und Erfahrungsraum, der sich demnach durch unterschiedliche Tätigkeitsfelder und Wirksamkeitserleben auszeichnet.

Das Engagement für FFF ist vorwiegend lokal verankert, findet in den jeweiligen Ortsgruppen statt und ist eng an lokale Gegebenheiten gebunden. Einen großen Stellenwert nimmt u.a. die ortsspezifische Vernetzung mit unterschiedlichen lokalen Gruppierungen ein. Zentrale Bedeutung haben dabei Kommunikations- und Aushandlungsprozesse, wobei die Auseinandersetzung und Begründung des eigenen Standpunktes in diskursiver Abgrenzung zu anderen Perspektiven gefordert ist. Die höhere intrinsische Motivation der aktiv Engagierten bestätigt vor dem Hintergrund bisheriger Studien (vgl. z.B. Schiefele \& Schreyer, 1994) Engagement als fruchtbaren Lern- und Bildungsraum.

Es zeigt sich, dass im Rahmen des Engagements Erfahrungen gemacht werden, die zu einer Veränderung der eigenen Selbstwirksamkeitserwartung führen könnten. Wenngleich die Analysen keine Aussagen zu kausalen Zusammenhängen erlauben, deuten die Gruppenvergleiche darauf hin, dass im aktiven Engagement für FFF-Bewegung, über die Teilnahme an Demonstrationen hinaus, auch Grenzen des eigenen Handelns und Wirkens erfahr- und spürbar werden, was sich in der niedrigeren Selbstwirksamkeitserwartung der Engagierten widerspiegeln kann. Die Unterschiede zwischen den verschiedenen Beteiligungsformen könnten die inkonsistenten Befunde von Wallis \& Loy (2021) zur Selbstwirksamkeitserwartung erklären. Während in ihren Untersuchungen die Selbstwirksamkeitserwartung und die Teilnahme an FFF-Protesten positiv miteinander korrelieren, zeigt sich die Selbstwirksamkeitserwartung in den multiplen Regressionen bei Konstanthaltung anderer Variablen als negativer Prädiktor für Umweltaktivismus.

Insgesamt wäre es für zukünftige Forschung zum Themenfeld aus bildungswissenschaftlicher Perspektive bedeutend, die Rolle der Schule und eine Verbindung von formalem und 
informellem Lernen angesichts der sozialökologischen Krise näher zu betrachten (vgl. dazu z.B. Budde, 2020). Schule und die darin agierenden Lehrkräfte können FFF als Chance rahmen (s. Beitrag Roncevic \& Pallesche in diesem Heft), gesellschaftsrelevante Inhalte und Fragestellungen kritisch aufzugreifen.

\section{Anmerkungen}

1 Aus Datenschutzgründen durften nur Personen im Alter von 16 Jahren und älter an der Umfrage teilnehmen

2 Neben den hier dargestellten Schwerpunkten wurden soziodemografische Merkmale, die Unterstützung durch das (u.a. schulische) Umfeld sowie Ängste (z.B. Klimawandel, Terroranschläge), geeigneten Maßnahmen gegen die Klimakrise und die Bedeutung verschiedener Ebenen (Politik, Individuum, Wirtschaft, soziale Bewegungen) erfasst.

\section{Literatur}

Bandura, A. (1997). Self-efficacy: The exercise of control. New York: Freeman.

Benedetti, S. (2015). Freiwilliges Engagement - ein bildungsbiografischer Erfahrungsraum. Zeitschrift Für Weiterbildungsforschung, 38(1), 53-69. https://doi. org/10.1007/s40955-015-0013-1

Braun, S. (2006). Soziale und politische Integration durch Vereine? Theoretische Ansätze und empirische Ergebnisse. In Soziale Ungleichheit, kulturelle Unterschiede Verhandlungen des 32 Kongresses der Deutschen Gesellschaft für Soziologie in München, Teilband 1 und 2 (S. 4498-4508). Frankfurt a. M.: Campus.

Braun-Wanke, K. \& Wagner, E. (2020). Über die Kunst, den Wandel zu gestalten. Kultur, Nachhaltigkeit, Bildung. Münster u. New York: Waxmann. https://doi. org/10.31244/9783830992868

Budde, J. (2020). Die Fridays for Future-Bewegung als Herausforderung für die Schule. Ein schulkritischer Essay. Die deutsche Schule, 112(2), 216-228. https://doi. org/10.31244/dds.2020.02.08

Deci, E. L. \& Ryan, R. M. (1993). Die Selbstbestimmungstheorie der Motivation und ihre Bedeutung für die Pädagogik. Zeitschrift für Pädagogik, 39(2), 223-238.

Döninghaus, L. M., et al. (2020). Kollektive Identität und kollektives Handeln. Wie werden Entscheidungen in Fridays for Future-Ortsgruppen getroffen? In S. Haunss \& M. Sommer (Hrsg.), Fridays for Future - Die Jugend gegen den Klimawandel (S. 147-162). Bielefeld: Transcript. https://doi.org/10.14361/9783839453476-007

Duguid, F., Mündel, K. \& Schugurensky, D. (2013). Volunteer Work and Informal Learning. In F. Duguid, K. Mündel \& D. Schugurensky (Hrsg.), Knowledge economy and education: Volunteer work, informal learning and social action (S. 17-36). Rotterdam: Sense Publishers. https://doi.org/10.1007/978-94-6209-233-4

Düx, W. (2018). Zivilgesellschaftliches Engagement. In K. Böllert (Hrsg.), Kompendium Kinder- und Jugendhilfe (S. 179-197). Wiesbaden: Springer VS. https:// doi.org/10.1007/978-3-531-19096-9_7

Düx, W. \& Sass, E. (2005). Lernen in informellen Kontexten. Lernpotenziale in Settings des freiwilligen Engagements. Zeitschrift für Erziehungswissenschaft, 8(3), 394-411. https://doi.org/10.1007/s11618-005-0147-9

Düx, W., Prein, G., Sass, E. \& Tully, C. J. (2009). Kompetenzerwerb im freiwilligen Engagement: Eine empirische Studie zum informellen Lernen im Jugendalter (2. Aufl., Schriften des Deutschen Jugendinstituts). Wiesbaden: VS Verlag für Sozialwissenschaften. https://doi.org/10.1007/978-3-531-91984-3

Elsdon, K. T., Reynolds, J. \& Stewart, S. (2002). Voluntary organisations: Citizenship, learning and change. Leicester: National Organisation for Adult Learning.

Goldenbaum, M. \& Thompson, C. S. (2020). Fridays for Future im Spiefel der Medienöffentlichkeit. In S. Haunss \& M. Sommer (Hrsg.), Fridays for Future - Die Jugend gegen den Klimawandel (S. 181-204). Bielefeld: Transcript. https://doi.org /10.14361/9783839453476-009

Göring, A. \& Mutz, M. (2016). Kompetenzerwerb und Persönlichkeitsentwicklung im Freiwilligen Sozialen Jahr - Ein Vergleich vier sozialer Tätigkeitsbereiche. Zeitschrift für Erziehungswissenschaft, 19(2), 395-414. https://doi.org/10.1007/s11618015-0632-8

Hanschmann, F. (2019). Fridays for Future: Versammlungsfreiheit vs. Schulpflicht. Ein Meinungsbild zur aktuellen Situation. Schulverwaltung Bayern, 42(7-8), 217 220 .

Hansen, S. (2008). Lernen durch freiwilliges Engagement: Eine empirische Studie zu Lernprozessen in Vereinen. Wiesbaden: VS Verlag für Sozialwissenschaften.

Haunss, S., Sommer, M. \& Fritz, L. (2020). Fridays for Future. Konturen einer neuen Protestbewegung. In S. Haunss \& M. Sommer (Hrsg.), Fridays for Future-
Die Jugend gegen den Klimawandel (S. 7-14). Bielefeld: Transcript. https://doi. org/10.14361/9783839453476-001

Holz, V., Kminek, H., Singer-Brodowski, M. \& Holfelder, A.-K. (2021). Erziehungswissenschaftliche Fragen im Zusammenhang mit der Bewegung Fridays for Future. Zeitschrift für Pädagogik, 67(1), 120-139.

Hurrelmann, K. \& Albrecht, E. (2020). Fridays for Future als Sinnbild ihrer Generation. In S. Haunss \& M. Sommer (Hrsg.), Fridays for Future - Die Jugend gegen den Klimawandel (S. 227-236). Bielefeld: Transcript. https://doi.org/10.14361/9 783839453476-011

Koos, S. \& Lauth, F. (2020). Die gesellschaftliche Unterstützung von Fridays for Future. In S. Haunss \& M. Sommer (Hrsg.), Fridays for Future - Die Jugend gegen den Klimawandel. Bielefeld: Transcript. https://doi.org/10.14361/9783839453476010

Koos, S. \& Lauth, F. (2019). Die Entwicklung und Zukunft der Fridays for Future-Bewegung. Zugriff am 08.09.2021 http://nbn-resolving.de/urn:nbn:de:bsz:35 2-2-1vspckwsvnogm8

Reinders, H. (2014). Jugend - Engagement - Politische Sozialisation: Gemeinnützige Tätigkeit und Entwicklung in der Adoleszenz. Wiesbaden: Springer VS. https://doi. org/10.1007/978-3-658-04628-6

Reuter, I. (2020). „Fridays for Future“ - über Klimaschwankungen im öffentlichen Diskurs angesichts des Widerstandes junger Menschen gegen den Totalitarismus der Sachzwänge. Zeitschrift für Pädagogik und Theologie, 72(3), 312-323. https:// doi.org/10.1515/zpt-2020-0035

Robertson, R. (1998). Glokalisierung, Homogenität und Heterogenität in Raum und Zeit. In U. Beck (Hrsg.), Perspektiven der Weltgesellschaft. (S. 192-220). Frankfurt a. M.: Suhrkamp.

Rucht, D. \& Ronk, D. (2020). Mobilisierungsprozesse von Fridays for Future. Ein Blick hinter die Kulissen. In S. Haunss \& M. Sommer (Hrsg.), Fridays for Future - Die Jugend gegen den Klimawandel (S.95-114). Bielefeld: Transcript. https://doi. org/10.14361/9783839453476-004

Schwarzer, R. \& Jerusalem, M. (Hrsg.) (1999). Skalen zur Erfassung von Lehrer-und Schülermerkmalen. Dokumentation der psychometrischen Verfahren im Rahmen der Wissenschaftlichen Begleitung des Modellversuchs Selbstwirksame Schulen. Berlin: Freie Universität Berlin.

Schiefele, U. \& Schreyer, I. (1994). Intrinsische Lernmotivation und Lernen: ein Überblick zu Ergebnissen der Forschung. Zeitschrift für Pädagogische Psychologie, 8(1), 1-13.

Sommer, M., Haunss, S., Gardner, B. G., Neuber, M. \& Rucht, D. (2020). Wer demonstriert da? Ergebnisse von Befragungen bei Großprotesten von Fridays for Future in Deutschland im März und November 2019. In S. Haunss \& M. Sommer (Hrsg.), Fridays for Future - Die Jugend gegen den Klimawandel (S. 15-66). Bielefeld: Transcript. https://doi.org/10.14361/9783839453476-002

Teune, S. (2020). Schulstreik. Geschichte einer Aktionsform und die Debatte über zivilen Ungehorsam. In S. Haunss \& M. Sommer (Hrsg.), Fridays for Future - Die Jugend gegen den Klimawandel (S. 131-146). Bielefeld: Transcript. https://doi. org/10.14361/9783839453476-006

Wahlström, M., Sommer, M., Kocyba, P. \& De Vydt, M. (2019). Protest for a future: Composition, mobilization and motives of the participants in Fridays For Future climate protests on 15 March, 2019 in 13 European cities. Zugriff am 02.08.2021 https://protestinstitut.eu/wp-content/uploads/2019/07/20190709_Protest-for-afuture_GCS-Descriptive-Report.pdf

Wallis, H. \& Loy, L. S. (2021). What drives pro-environmental activism of young people? A survey study on the Fridays For Future movement. Journal of Environmental Psychology, 74. https://doi.org/10.1016/j.jenvp.2021.101581

\section{Dr. Jana Costa}

Wissenschaftliche Mitarbeiterin im Arbeitsbereich Lernumwelten und schulische Bildung am Leibniz Institut für Bildungsverläufe. Arbeits- und Forschungsschwerpunkte: Bildung für nachhaltige Entwicklung, Freiwilliges Engagement als Lern- und Erfahrungsraum, Transformations- und Innovationsprozesse in universitärer Lehre, Mixed-Methods-Designs in der empirischen Bildungsforschung.

\section{Elena Wittmann}

Wissenschaftliche Mitarbeiterin im Arbeitsbereich Persönlichkeit am Leibniz Institut für Bildungsverläufe. Arbeits- und Forschungsschwerpunkte: sozialund persönlichkeitspsychologische Mechanismen bei Bildungsverläufen, Geschwisterforschung, soziale Ungleichheit. 